\title{
Effect of Two Temperatures and Thermal Phase-lags in a Thick Plate due to a Ring Load with Axisymmetric Heat Supply
}

\author{
Rajneesh Kumar ${ }^{1}$, Nidhi Sharma ${ }^{2}$, Parveen Lata ${ }^{3 *}$ \\ ${ }^{1}$ Department of Mathematics, Kurukshetra University \\ Kurukshetra, Haryana, India \\ E-mail: rajneesh_kuk@rediffmail.com \\ ${ }^{2}$ Department of Mathematics, MM University \\ Mullana, Ambala, Haryana, India \\ E-mail: nidhi_kuk26@rediffmail.com \\ ${ }^{3}$ Department of Basic and Applied Sciences, Punjabi University \\ Patiala, Punjab, India \\ *E-mail: parveenlata@pbi.ac.in
}

Received: 31 Janurary 2016; revised: 22 September 2016; accepted: 26 September 2016; published online: 30 September 2016

\begin{abstract}
The present investigation concerns thermomechanical interactions in a homogeneous isotropic thick plate in the light of the two-temperature thermoelasticity theory with dual phase lag due to a ring load. The upper and lower ends of the thick plate are traction free and subjected to an axisymmetric heat supply. The solution is obtained by using Laplace and Hankel transform techniques. The analytical expressions of displacement components, stresses, conductive temperature, temperature change and cubic dilatation are computed in a transformed domain. The numerical inversion technique has been applied to obtain the results in the physical domain. Numerically simulated results are depicted graphically. The effect of thermal phase-lags and two temperatures are shown on the various components. Some particular cases of the result are also deduced from the present investigation.
\end{abstract}

Key words: two-temperature, two phase lags, isotropic, ring load, Laplace transform, Hankel transform

\section{INTRODUCTION}

Classical Fourier heat conduction law implies an infinitely fast propagation of a thermal signal which is violated in the ultra-fast heat conduction system due to its very small dimensions and short time scales. Catteno [1] and Vernotte [2] proposed a thermal wave with a single phase lag in which the temperature gradient after a certain elapsed time was given by $\mathbf{q}+\tau_{q} \frac{\partial \mathbf{q}}{\partial t}=-k \nabla T$, where $\tau_{q}$ denotes the relaxation time required for thermal physics to take account of a hyperbolic effect within the medium. Here when $\tau_{q}>0$, the thermal wave propagates through the medium with a finite speed of $\sqrt{\alpha / \tau_{q}}$, where $\alpha$ is thermal diffusivity. when $\tau_{q}$ approaches zero, the thermal wave has an infinite speed and thus the single phase lag model reduces to the traditional Fourier model. The dual phase lag model of heat conduction was proposed by [3] $\mathbf{q}+\tau_{q} \frac{\partial \mathbf{q}}{\partial t}=-k\left(\nabla T+\tau_{t} \frac{\partial}{\partial t} \nabla T\right)$, where the temperature gradient $\nabla T$ at a point $P$ of the material at time $t+\tau_{t}$ corresponds to the heat flux vector $q$ at the same time at the time $t+\tau_{q}$. Here $k$ is thermal conductivity of the material. The delay time $\tau_{t}$ is interpreted as that caused by the microstructural interactions and is called the phase lag of temperature gradient. The other delay time $\tau_{q}$ is interpreted as the relaxation time due to the fast transient effects of thermal 
inertia and is called the phase lag of heat flux. This universal model is claimed to be able to bridge the gap between microscopic and macroscopic approaches, covering a wide range of heat transfer models. If $\tau_{t}=0$, [3] refers to the model as a single phase model. Numerous efforts have been invested in the development of an explicit mathematical solution to the heat conduction equation under a dual phase lag model. Quintanilla [4] compared two different mathematical hyperbolic models proposed by Tzou. Kumar and Mukhopadhaya [5] investigated the propagation of harmonic waves of assigned frequency by employing the thermoelasticity theory with three phase lags. Chou and Yang [6] discussed two dimensional dual phase lag thermal behaviour in single-/multi layer structures. Zhou, Zhang and Chen [7] proposed an axisymmetric dual-phase-lag bioheat model for laser heating of living tissues. Kumar, Chawla and Abbas [8] discussed the effect of viscosity on wave propagation in an anisotropic thermoelastic medium with a three phase lag model. Ying and Yun [9] built a fractional dual-phase-lag model and the corresponding bio-heat transfer equation. Abdallah [10] used an uncoupled thermoelastic model based on a dual phase lag to investigate the thermoelastic properties of a semi infinite medium. Rukolaine [11] employed dual phase lag models to study unphysical problems. Tripathi, Kedar and Deshmukh [12] discussed the generalized thermoelastic diffusion problem in a thick circular plate with axisymmetric heat supply.

Chen and Gurtin [13], Chen et al. [14] and Chen et al. [15] have formulated a theory of heat conduction in deformable bodies which depends upon two distinct temperatures, the conductive temperature $\phi$ and the thermo dynamical temperature $T$. For time independent situations the difference between these two temperatures is proportional to the heat supply, and in the absence of heat supply the two temperatures are identical. For time dependent problems the two temperatures are different regardless of the presence of heat supply. The two temperatures $T, \phi$ and the strain are found to have representations in the form of a travelling wave plus a response, which occurs instantaneously throughout the body (Boley and Tolins [16]).The wave propagation in the two temperature theory of thermoelasticity was investigated by Warren and Chen [17]. Youssef [18], constructed a new theory of generalized thermoelasticity by taking into account the two-temperature generalized thermoelasticity theory for a homogeneous and isotropic body without energy dissipation. Several researchers studied various problems involving two temperature, e.g. ( [19-27].

Many researchers havealready worked on the problems of dual phase lags with two temperatures considering different mediums. Mondal, Malik and Kanoria [28] investigated a problem of fractional order two temperature dual phase lag thermoelasticity with variable thermal conductivity. Said [29] discussed deformation of a rotating two temperature generalized magneto thermoelastic medium with an initial heat source due to hydrostatic initial stress. Said [30] studied two temperature generalized magneto thermoelastic medium for dual phase lag model under the effect of a gravity field and hydrostatic initial stress. Lofty [31] worked on the elastic wave motions for a photothermal medium of a dual phase lag model with an internal heat source and gravitational field.

In this investigation, the thermoelastic interactions for the dual phase lag heat conduction in a thick circular plate due to a ring load is studied in the light of the two temperature thermoelasticity theory. The components of displacements, stresses, conductive temperature, temperature change and cubic dilatation are computed numerically. Numerically computed results are depicted graphically. The effect of dual phase lag and two temperature are shown on the various components.

\section{BASIC EQUATIONS}

The basic equations of motion, heat conduction in a homogeneous isotropic thermoelastic solid with dual phase lag and two temperature in the absence of body forces, heat sources are

$$
(\lambda+\mu) \nabla(\nabla . u)+\mu \nabla^{2} u-\beta_{1} \nabla T=\rho \ddot{u}
$$

$$
\begin{gathered}
\left(1+\tau_{t} \frac{\partial}{\partial t}\right) K T_{, i i}= \\
=\left(1+\tau_{q} \frac{\partial}{\partial t}+\tau_{q}{ }^{2} \frac{\partial^{2}}{\partial t^{2}}\right)\left[\rho C_{E} \dot{T}+\beta_{1} T_{0} \dot{e}_{k k}\right] \\
T=\left(1-a \nabla^{2}\right) \phi
\end{gathered}
$$

and the constitutive relations are

$$
\begin{gathered}
\sigma_{i j}=2 \mu e_{i j}+\delta_{i j}\left(\lambda e_{k k}-\beta_{1} T\right) \\
\rho T_{0} S=\left(1+\tau_{q} \frac{\partial}{\partial t}+\tau_{q}{ }^{2} \frac{\partial^{2}}{\partial t^{2}}\right)\left(\rho C_{E} T+\beta_{1} T_{0} e_{k k}\right)
\end{gathered}
$$

Where $\lambda, \mu$ are Lame's constants, $\rho$ is the density assumed to be independent of time, $u_{i}$ are components of displacement vector $u, K$ is the coefficient of thermal conductivity, $C_{E}$ is the specific heat at constant strain, $T$ is the absolute temperature of the medium, $\sigma_{i j}$ and $e_{i j}$ are the components of stress and strain, respectively, $e_{k k}$ is dilatation, $S$ is the entropy per unit mass, $\beta_{1}=(3 \lambda+2 \mu) \alpha_{t}, \alpha_{t}$ is the coefficient of thermal linear expansion. $\tau_{t}, \tau_{q}$ are respectively the phase lag of temperature gradient, the phase lag of heat flux, $a$ is the two temperature parameter. In the above equations, a comma followed by suffix denotes a spatial derivative and a superposed dot denotes a derivative with respect to time. 


\section{FORMULATION AND SOLUTION OF THE PROBLEM}

Consider a homogeneous isotropic thick plate of thickness $2 b$ occupying the space defined by $0 \leq r \leq \infty,-b \leq z \leq b$. Cylindrical polar coordinates $(r, \theta, z)$ having origin on the surface $z=0$, between the lower and upper surfaces of the plate and the $z$-axis is assumed to be the axis of symmetry. Due to symmetry about $z$-axis, component $u_{\theta}=0$, and $u_{r}, u_{z}$ and $\phi$ are independent of $\theta$ and are functions of $(r, z, t)$. The initial temperature in the thick plate is given by a constant temperature $T_{0}$, and the heat flux $g_{0} F(r, z)$ is prescribed on the upper and lower boundary surfaces. For $t>0$, heat is generated within the plate at the rate $f(r, t)$. We consider a normal source (ring source) which emanates from the origin of the coordinate axis and expands radically at constant rate $c$ over the surface. Under these conditions, thermoelastic quantities due to the ring load are to be determined. As the problem considered is two dimensional,

$$
\mathbf{u}=\left(u_{r}, 0, u_{z}\right)
$$

Equations (1)-(2) with the aid of (5) take the form

$$
\begin{gathered}
(\lambda+\mu) \frac{\partial e}{\partial r}+\mu\left(\nabla^{2}-\frac{1}{r^{2}}\right) u_{r}-\beta_{1} \frac{\partial T}{\partial r}=\rho \frac{\partial^{2} u_{r}}{\partial t^{2}} \\
(\lambda+\mu) \frac{\partial e}{\partial z}+\mu \nabla^{2} u_{z}-\beta_{1} \frac{\partial T}{\partial z}=\rho \frac{\partial^{2} u_{z}}{\partial t^{2}} \\
\left(1+\tau_{t} \frac{\partial}{\partial t}\right) K \nabla^{2} T=\left(1+\tau_{q} \frac{\partial}{\partial t}+\frac{\tau_{q}^{2}}{2} \frac{\partial^{2}}{\partial t^{2}}\right) \times \\
{\left[\rho C_{E} \frac{\partial}{\partial t}\left(1-a \nabla^{2}\right) \phi+\beta_{1} T_{0} \frac{\partial}{\partial t} \operatorname{div} u\right]}
\end{gathered}
$$

and Constitutive relations

$$
\begin{gathered}
\sigma_{r r}=2 \mu e_{r r}+\lambda e-\beta_{1}\left(1-a \nabla^{2}\right) \phi \\
\sigma_{\theta \theta}=2 \mu e_{\theta \theta}+\lambda e-\beta_{1}\left(1-a \nabla^{2}\right) \phi \\
\sigma_{z z}=2 \mu e_{z z}+\lambda e-\beta_{1}\left(1-a \nabla^{2}\right) \phi \\
\sigma_{r z}=\mu e_{r z}, \sigma_{r \theta}=0, \sigma_{z \theta}=0,
\end{gathered}
$$

where $e=\frac{\partial u_{r}}{\partial r}+\frac{u_{r}}{r}+\frac{\partial u_{z}}{\partial z}, e_{r r}=\frac{\partial u_{r}}{\partial r}, e_{\theta \theta}=\frac{u_{r}}{r}, e_{z z}=\frac{\partial u_{z}}{\partial z}$, $e_{r z}=\frac{1}{2}\left(\frac{\partial u_{r}}{\partial z}+\frac{\partial u_{z}}{\partial r}\right)$
To facilitate the solution, the following dimensionless quantities are introduced

$$
\begin{aligned}
& r^{\prime}=\frac{\omega_{1}}{c_{1}} r, z^{\prime}=\frac{\omega_{1}}{c_{1}} z,\left(u_{r}^{\prime}, u_{z}^{\prime}\right)=\frac{\omega_{1}}{c_{1}}\left(u_{r}, u_{z}\right), t^{\prime}=\omega_{1} t, \\
& \begin{aligned}
\omega_{1} & =\frac{\rho C_{E} c_{1}^{2}}{K}, c_{1}^{2}=\frac{\lambda+2 \mu}{\rho}\left(\sigma_{r r}^{\prime}, \sigma_{\theta \theta}^{\prime}, \sigma_{z z}^{\prime}, \sigma_{r z}^{\prime}\right)= \\
& =\frac{1}{\beta_{1} T_{0}}\left(\sigma_{r r}, \sigma_{\theta \theta}, \sigma_{z z}, \sigma_{r z}\right),
\end{aligned} \\
& \left(T^{\prime}, \phi^{\prime}\right)=\frac{\beta_{1}}{\rho c_{1}^{2}}(T, \phi)\left(\tau_{q}^{\prime}, \tau_{t}^{\prime}\right)=\omega_{1}\left(\tau_{q}, \tau_{t}\right)
\end{aligned}
$$

We define Laplace and Hankel transform as

$$
\begin{gathered}
\bar{f}(r, z, s)=\int_{0}^{\infty} f(r, z, t) e^{-s t} d t \\
\bar{f}^{*}(\xi, z, s)=\int_{0}^{\infty} \hat{f}(r, z, s) r J_{n}(r \xi) d r
\end{gathered}
$$

Using the dimensionless quantities defined by (13) in equations (6)-(8) and suppressing the primes for convenience and applying the Laplace transform defined by (14) on the resulting equations and simplifying, we obtain

$$
\begin{gathered}
\left(\nabla^{2}-\mathrm{s}^{2}\right) \bar{e}-\nabla^{2} \bar{\phi}+\delta_{1} \nabla^{4} \bar{\phi}=0 \\
\tau_{q}^{1} \zeta_{2} \bar{e}+\tau_{q}^{1} \zeta_{1} \bar{\phi}-\left(\tau_{q}^{1} \delta_{1}-\tau_{t}^{1} K\right) \nabla^{2} \bar{\phi}=0
\end{gathered}
$$

where $\tau_{q}^{1}=1+s \tau_{q}+\frac{s^{2} \tau_{q}{ }^{2}}{2}, \tau_{t}^{1}=1+s \tau_{t}, \zeta_{1}=\frac{\rho C_{E} c_{1}^{2}}{\omega_{1}}$, $\zeta_{2}=\frac{\beta_{1}^{2} T_{0}}{\rho \omega_{1}} s, \delta_{1}=\frac{a \omega_{1}^{2}}{c_{1}^{2}}$

Eliminating $\bar{\phi}$ and $\bar{e}$ from equations (16)-(17), we obtain

$$
\left(\nabla^{2}-k_{1}^{2}\right)\left(\nabla^{2}-k_{2}^{2}\right)(\bar{e}, \bar{\phi})=0
$$

The solutions of the equation (18) can be written in the form $\bar{\phi}=\sum_{i=1}^{3} \bar{\phi}_{i}, \bar{e}=\sum_{i=1}^{3} \bar{e}_{i}$ where, $\bar{e}_{i}$, and $\bar{\phi}_{i}$ are solutions of the following equation

$$
\left(\nabla^{2}-k_{i}^{2}\right)\left(\bar{e}_{i}, \bar{\phi}_{i}\right)=0, i=1,2
$$

On taking Hankel transform of (19) defined by (15), we obtain

$$
\left(D^{2}-\xi^{2}-k_{i}^{2}\right)\left(\bar{\phi}_{i}^{*}, \bar{e}_{i}^{*}\right)=0
$$

The solution of (20) has the form

$$
\begin{gathered}
\bar{e}^{*}=\sum_{i=1}^{2} A_{i}(\xi, s) \cosh \left(q_{i} z\right) \\
\bar{\phi}^{*}=\sum_{i=1}^{2} d_{i} A_{i}(\xi, s) \cosh \left(q_{i} z\right)
\end{gathered}
$$

where $q_{i}=\sqrt{\xi^{2}+k_{i}^{2}}, d_{i}=\frac{\tau_{q}^{1} \zeta_{2}}{\tau_{q}^{1} \zeta_{1}-\zeta_{3} q_{i}^{2}}, \zeta_{3}=\tau_{q}^{1} \delta_{1}-\tau_{t}^{1} k$. 
We define the inversion of Hankel Transform as

$$
\bar{f}(r, z, s)=\int_{0}^{\infty} \bar{f}^{*}(\xi, z, s) \xi J_{0}(r \xi) d \xi
$$

Applying inversion of Hankel transform defined by (23) on the equations (21)-(22), we obtain

$$
\begin{aligned}
& \bar{e}=\int_{0}^{\infty}\left\{\sum_{i=1}^{2} A_{i}(\xi, s) \cosh \left(q_{i} z\right)\right\} \xi J_{0}(\xi r) d \xi \\
& \bar{u}_{r}(r, z, s)=\int_{0}^{\infty} E(\xi, s) \cosh (q z) \xi J_{0}(\xi r)+\sum_{i=1}^{2}\left[\left(-\eta_{i}+\mu_{i}\right) q_{i}^{2} \xi^{2} \cosh \left(q_{i} z\right)\right] J_{1}(\xi r)+ \\
& \left.\delta_{1} \mu_{i} \cosh \left(q_{i} z\right)\left(\frac{\xi^{3}}{r} J_{2}-J_{1}\left(\xi^{4}-\frac{\xi^{2}}{r^{2}}+\xi^{2} q_{i}^{2}\right)+\frac{\xi^{3}}{r} J_{0}\right)\right] d \xi \\
& \bar{u}_{z}(r, z, s)=\int_{0}^{\infty} F(\xi, z) \sinh (q z) \xi J_{0}(\xi r)+\sum_{i=1}^{2}\left[\left(-\eta_{i}+\mu_{i}\right) \sinh \left(q_{i} z\right) \xi J_{0}(\xi r)-\right. \\
& \left.\delta_{1} \mu_{i} \sinh \left(q_{i} z\right)\left(\frac{\xi^{2}}{r} J_{2}-J_{1}\left(\xi^{3}+\frac{\xi}{r}\right)+\xi q_{i}^{2} J_{0}\right)\right] d \xi \\
& \sigma_{z z}^{-}=\frac{2 \mu}{\beta_{1} T_{0}} \int_{0}^{\infty} \xi J_{0}(\xi r)\left[F(\xi, s) q \cosh (q z)+\left(\sum_{i=1}^{2}\left(-\eta_{i}+\mu_{i}\right) q_{i}^{2}-\zeta d_{i} \mathrm{~A}_{\mathrm{i}}+\lambda^{0} \mathrm{~A}_{\mathrm{i}}\right) \cosh \left(q_{i} z\right)\right]- \\
& {\left[\delta_{1}\left(\mu_{i} q_{i}-\zeta B_{i}\right) \cosh \left(q_{i} z\right)\left(\xi^{3} J_{0}(\xi r)-J_{1}(\xi r)\left(\frac{\xi-1}{r}\right)+\xi q_{i}^{2} J_{0}(\xi r)\right] d \xi\right.} \\
& \sigma_{r z}^{-}=\frac{\mu}{2 \beta_{1} T_{0}} \int_{0}^{\infty} \xi^{2} J_{1}(\xi r)\left[\left\{\left(\frac{q^{2}-\xi^{2}}{q}\right) E(\xi, s) q \sinh (q z)+2 \sum_{i=1}^{2}\left(\eta_{i}-\mu_{i}\right) q_{i} \sinh \left(q_{i} z\right)+\right.\right. \\
& \left.\delta_{1} \mu_{i} \sinh \left(q_{i} z\right)\left\{\left(q_{i}\left(\frac{\xi^{3}}{r} J_{2}(\xi r)\right)\right\}-J_{1}(\xi r)\left(\xi^{4}+q_{i}^{2} \xi^{2}+\frac{4}{\xi^{2} r^{2}}+q_{i}^{2} \xi\right)+\left(\frac{2}{\xi r}-\xi^{4}-\frac{\xi^{2}(\xi-1)}{r}+q_{i}^{2} \xi^{2}\right) J_{0}(\xi r)\right\}\right] d \xi \\
& \sigma_{r r}^{-}=\frac{2 \mu}{\beta_{1} T_{0}} \int_{0}^{\infty}\left[-\xi^{2} J_{1}(\xi r) E(\xi, s) \cosh (q z)+\left(\sum_{i=1}^{2}\left(-\eta_{i}+\mu_{i}\right) q_{i}^{2}\left(\xi^{2} J_{1}(\xi r)-\xi^{3} J_{0}(\xi r)\right)+\right.\right. \\
& \delta_{1} \mu_{i}\left\{J_{1}(\xi r)\left(-\xi^{2}\left(q_{i}^{2}+\xi^{2}-3\right)+J_{0}(\xi r) \xi^{3}\left(q_{i}^{2}+\frac{1}{r^{2}}+\xi^{2}\right) \mathrm{d} \xi\right]+\right. \\
& \int_{0}^{\infty} \xi J_{0}(\xi r) \cosh \left(q_{i} z\right)\left[\lambda^{0}-\zeta d_{i}\left(1+\delta_{1} q_{i}^{2}-\delta_{1} \xi J_{0}(\xi r)\right)+\delta_{1} J_{1}(\xi r)\left(1-\frac{1}{r}\right) d \xi\right. \\
& \bar{\phi}=\int_{0}^{\infty}\left(d_{1} A_{1}(\xi, s) \cosh \left(q_{1} z\right)+d_{2} A_{2}(\xi, s) \cosh \left(q_{2} z\right)\right) \xi J_{0}(\xi r) d \xi \\
& \bar{T}=\int_{0}^{\infty} \sum_{i=1}^{2}\left(d_{i} A_{i}(\xi, s) \cosh \left(q_{i} z\right)\left[\xi J_{0}(\xi r)\left(1+\delta_{1} q_{i}^{2}-\delta_{1} \xi\right)+J_{1}(\xi r) \delta_{1} \xi\left(1-\frac{1}{r}\right)\right] d \xi,\right.
\end{aligned}
$$


where

$$
\begin{gathered}
G(\xi, s)=\frac{\xi^{2} E(\xi, s)}{q}, q=\sqrt{\xi^{2}+\frac{\rho c_{1}^{2}}{\mu} s^{2}}, \eta_{i}=\frac{\frac{\lambda+\mu}{\rho c_{1}^{2}} A_{i}}{\left(\frac{\mu q_{i}^{2}}{\rho c_{1}^{2}}-s^{2}\right)}, \mu_{i}=\frac{d_{i} A_{i}}{\left(\frac{\mu q_{i}^{2}}{\rho c_{1}^{2}}-s^{2}\right)}, \\
\lambda^{0}=\frac{\lambda}{\beta_{1} T_{0}}, \zeta=\frac{\rho c_{1}^{2}}{\beta_{1} T_{0}} .
\end{gathered}
$$

\section{BOUNDARY CONDITIONS}

We consider a cubical thermal source and normal force of unit magnitude along with vanishing of tangential stress components at the stress free surface at $z= \pm b$. Mathematically, these can be written as

$$
\begin{gathered}
\frac{\partial \phi}{\partial z}= \pm g_{0} F(r, z) \\
\sigma_{z z}=f(r, t) \\
\sigma_{r z}=0
\end{gathered}
$$

\section{APPLICATION}

As an application, we consider a specific type of source function of the type

$$
\begin{gathered}
F(r, z)=z^{2} e^{-\omega r} \\
f(r, t)=\frac{1}{2 \pi r} \delta(t-r),
\end{gathered}
$$

where $\delta(t-r)$ is the Dirac delta function.

Applying Laplace transform and Hankel transform defined by (14)-(15), on the equations (35)-(36), we obtain

$$
\begin{aligned}
& \bar{F}^{*}(\xi, z)=\frac{z^{2} \omega}{\left(\xi^{2}+\omega^{2}\right)^{3 / 2}}, \\
& \bar{f}^{*}(\xi, s)=\frac{1}{2 \pi \sqrt{\xi^{2}+\frac{s^{2}}{c^{2}}}} .
\end{aligned}
$$

Applying Laplace transform and Hankel transform on the boundary conditions (32)-(34), we obtain and substitute the values of $\bar{\phi}, \bar{\sigma}_{z z}, \bar{\sigma}_{r z}$, in (34)-(36), we obtain the values of unknown parameters as

$$
\begin{gathered}
A_{1}=\frac{\triangle_{1}}{\triangle}, A_{2}=\frac{\triangle_{2}}{\triangle}, E(\xi, s)=\frac{\triangle_{3}}{\triangle}, \text { where } \\
\triangle \\
\sinh (q b)\left(\triangle_{11} \triangle_{22}-\triangle_{12} \triangle_{21}\right) \\
\triangle_{1} T_{0}=g_{0} \bar{F}^{*}(\xi, z)\left(\triangle_{21} \triangle_{32}-\triangle_{22} \triangle_{31}\right)- \\
\bar{f}^{*}(\xi, s)\left(\triangle_{11} \triangle_{32}-\triangle_{12} \triangle_{31}\right) \\
\triangle_{2}=-g_{0} \bar{F}^{*}(\xi, z)\left(\frac{2 \mu}{\beta_{1} T_{0}} \cosh (q b) \triangle_{32}-\right. \\
\left.\triangle_{22} \sinh (q b)\right)+\bar{f}^{*}(\xi, s)\left(-\triangle_{12} \sinh (q b)\right)
\end{gathered}
$$

$$
\begin{aligned}
& \triangle_{3}=g_{0} \bar{F}^{*}(\xi, z)\left(\frac{2 \mu}{\beta_{1} T_{0}} \cosh (q b) \triangle_{31}-\right. \\
& \left.\triangle_{21} \sinh (q b)\right)+\bar{f}^{*}(\xi, s)\left(\triangle_{11} \sinh (q b)\right)
\end{aligned}
$$

$\triangle_{1 i}=d_{i} q_{i} \sinh \left(q_{i} b\right), \triangle_{2 i}=\left(\left(\mu_{i}-\eta_{i}\right) q_{i}^{2}-\delta_{1} \mu_{i} q_{i}-\right.$ $\left.\zeta d_{i}\left(1+\delta_{1}\right)+\lambda^{\prime}\right) \cosh \left(q_{i} b\right), \triangle_{3 i}=\left(2\left(\eta_{i}-\mu_{i}\right) q_{i}+\delta_{1} \mu_{i}\right) \times$ $\sinh \left(q_{i} b\right), i=1,2 . \bar{F}^{*}(\xi, z)$ and $\bar{f}^{*}(\xi, s)$ are given by equations (37)-(38).

\section{PARTICULAR CASES}

(i) If $a=0$, from equations (25)-(30), we obtain the corresponding expressions for displacements, and stresses, conductive temperature, temperature change and cubic dilatation for thermoelastic solid without two temperature and due to a dual phase lag.

(ii) If $\tau_{q}=\tau_{t}=0$, we obtain the coupled expression in thermoelasticity with a two temperature model.

(iii) $\tau_{q}=0$ then dual phase lag thermal model (DPLT) model reduce to single-phase-lag thermal model (SPLT) 


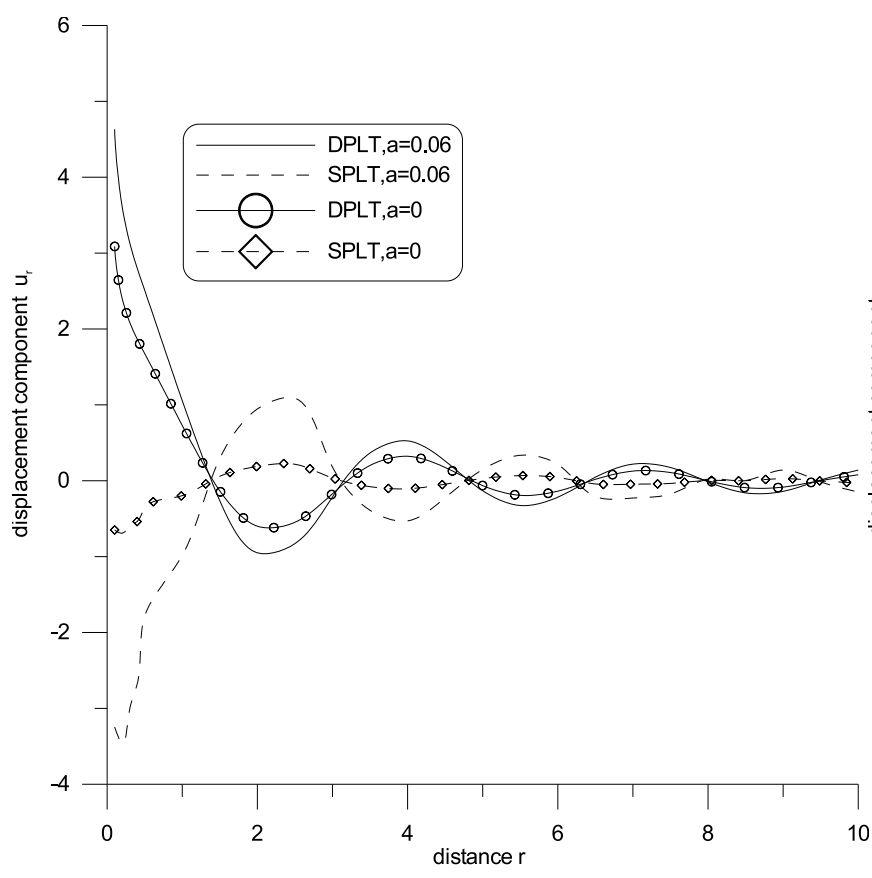

Fig. 1. Variation of displacement component $u_{r}$ with distance $r$

\section{INVERSION OF DOUBLE TRANSFORM}

Due to the complexity of the solution in the Laplace transform domain, the inverse of the Laplace transform is obtained by using the Gaver-Stehfast algorithm. [32-34] derived the formula given below. By this method, the inverse $f(t)$ of Laplace transform $\bar{f}(s)$ is approximated by

$$
f(t)=\frac{\log 2}{t} \sum_{j=1}^{k} D(j, K) F\left(j \frac{\log 2}{t}\right)
$$

with

$$
\begin{aligned}
& D(j, K)=(-1)^{j+M} \times \\
& \sum_{n=m}^{\min (j, M)} \frac{n^{M}(2 n) !}{(M-n) ! n !(n-1) !(j-n) !(2 n-j) !},
\end{aligned}
$$

Where $K$ is an even integer, whose value depends on the word length of the computer used. $M=K / 2$ and $m$ is an integer part of $(j+1) / 2$. The optimal value of $K$ was chosen as described in the Gaver-Stehfast algorithm for fast convergence of results with desired accuracy. The Romberg numerical integration technique (Press et. al. [35]) with variable step size was used to evaluate the results involved.

\section{NUMERICAL RESULTS AND DISCUSSION}

The mathematical model is prepared with copper material for purposes of numerical computation. The mate-

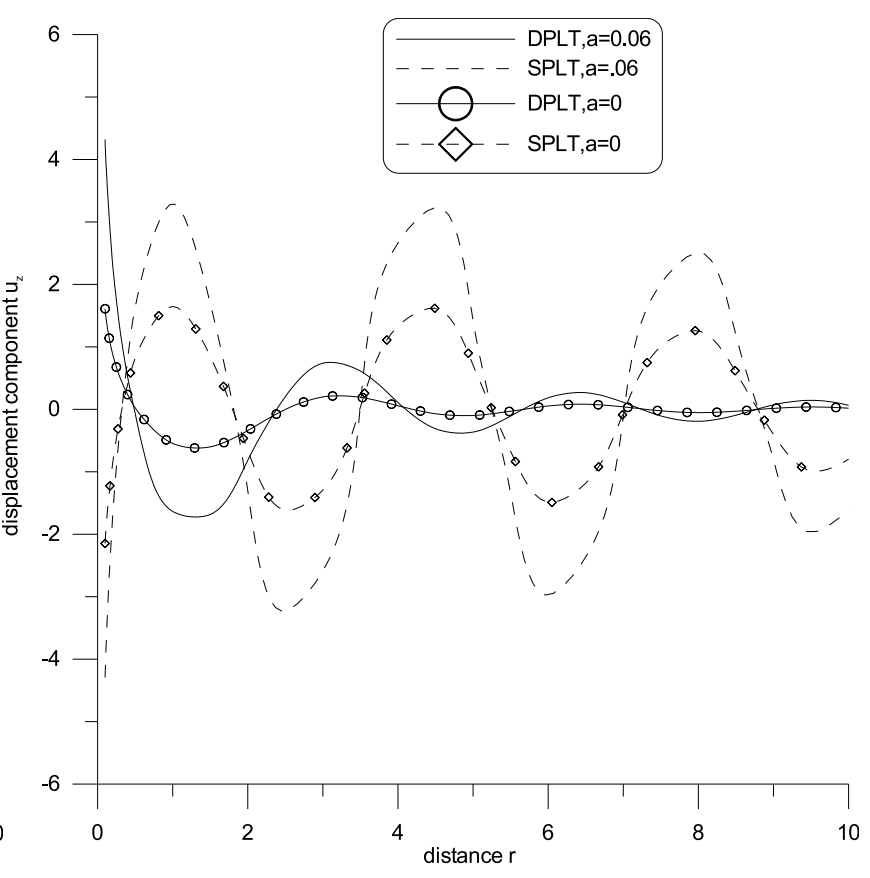

Fig. 2. Variation of displacement component $u_{z}$ with distance $r$

rial constants for the problem are taken from Dhaliwal and Singh [36].

$$
\begin{aligned}
& \lambda=7.76 \times 10^{10} \mathrm{Nm}^{-2}, \\
& \mu=3.86 \times 10^{10} \mathrm{Nm}^{-2}, \\
& K=386 J \mathrm{~J}^{-1} \mathrm{~m}^{-1} \mathrm{~s}^{-1}, \\
& \beta_{1}=5.518 \times 10^{6} \mathrm{Nm}^{-2} \mathrm{deg}^{-1}, \\
& \rho=8954 \mathrm{Kg} \mathrm{m}^{-3}, \\
& a=1.2 \times 10^{4} \mathrm{~m}^{2} / \mathrm{s}^{2} k, \\
& b=0.9 \times 10^{6} \mathrm{~m}^{5} / \mathrm{kg} \mathrm{s}^{2}, \\
& D=0.88 \times 10^{-8} \mathrm{~kg} \mathrm{~s}^{3} \mathrm{~m}^{3}, \\
& \beta_{2}=61.38 \times 10^{6} \mathrm{Nm}^{-2} \mathrm{deg}^{-1} \\
& T_{0}=293 \mathrm{~K}, \\
& C_{E}=383.1 \mathrm{~J} \mathrm{~kg}^{-1} \mathrm{~K}^{-1}
\end{aligned}
$$

The graphs have been plotted to study the effect of two temperatures on a dual phase lag thermal (DPLT) model and a single phase lag thermal (SPLT) model on the various quantities in the range $0 \leq r \leq 10$.

1. In the figures th solid line corresponds to the dualphase-lag of heat transfer with two temperature (DPLT, $a=.06$ ).

2. The small dashed line corresponds to the single phase-lag of heat transfer with two temperature (SPLT, $a=.06)$. 


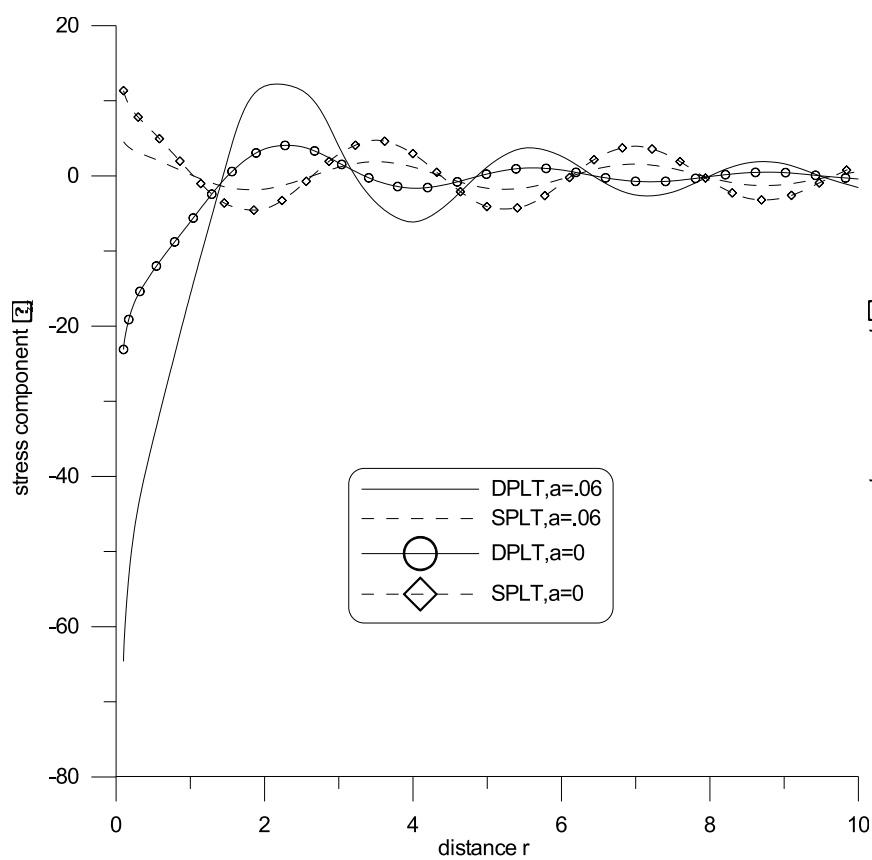

Fig. 3. Variation of stress component $\sigma_{z z}$ with distance $r$

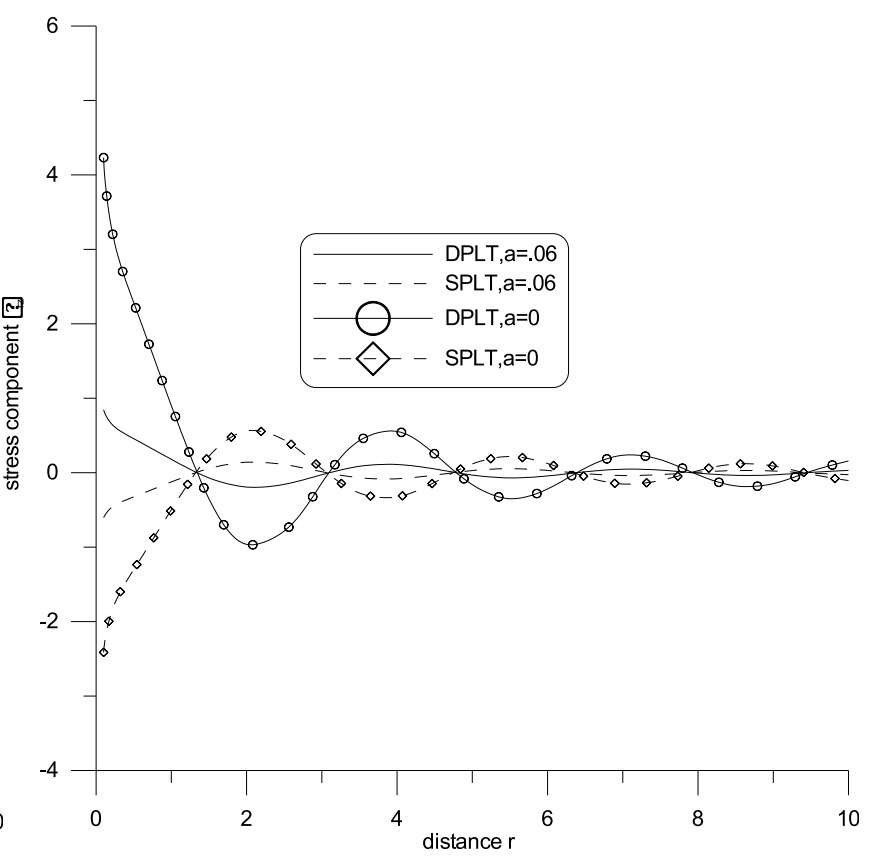

Fig. 4. Variations of stress component $\sigma_{r z}$ with distance $r$

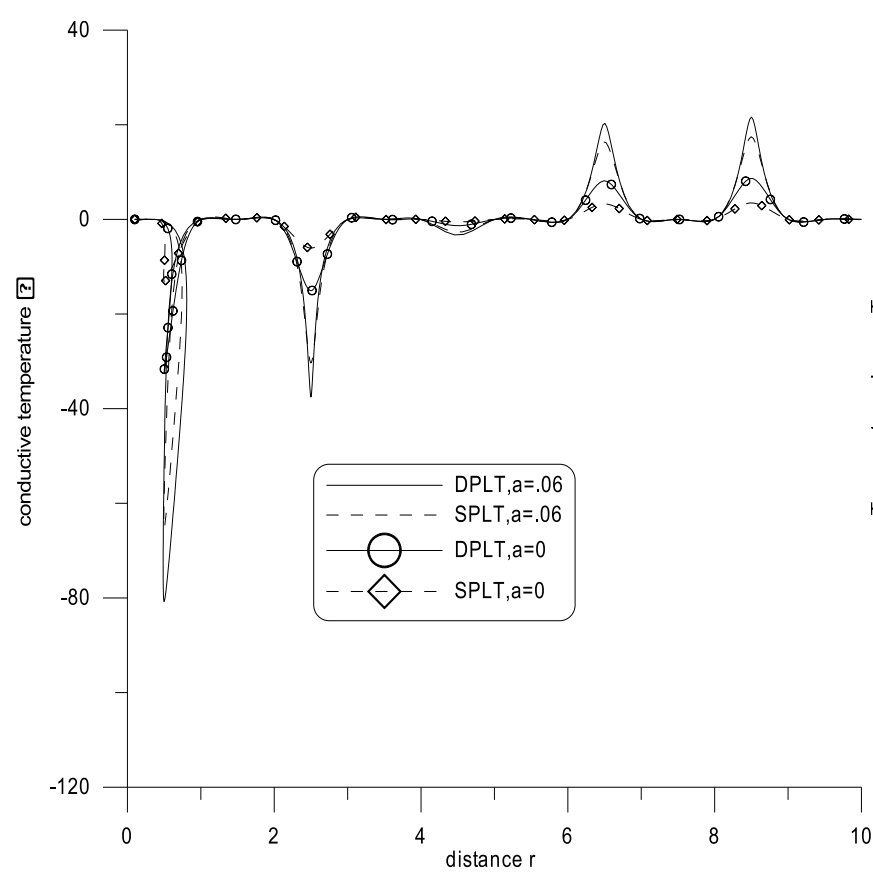

Fig. 5. Variation of conductive temperature $\phi$ with distance $r$

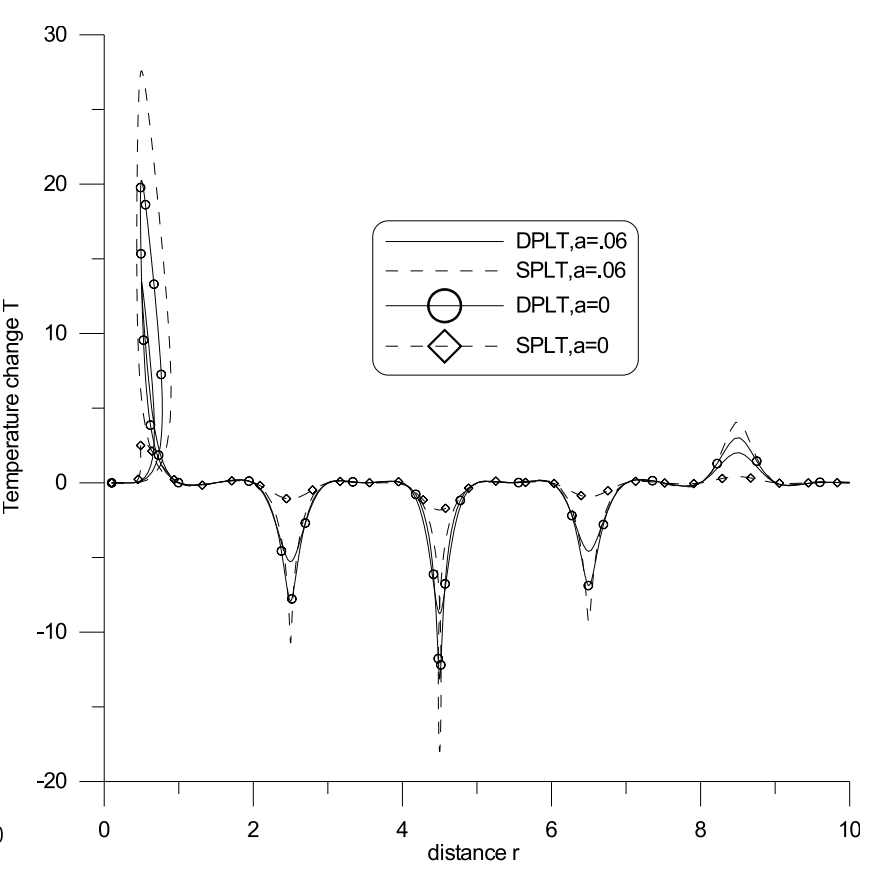

Fig. 6. Variations of temperature change $T$ with distance $r$ 


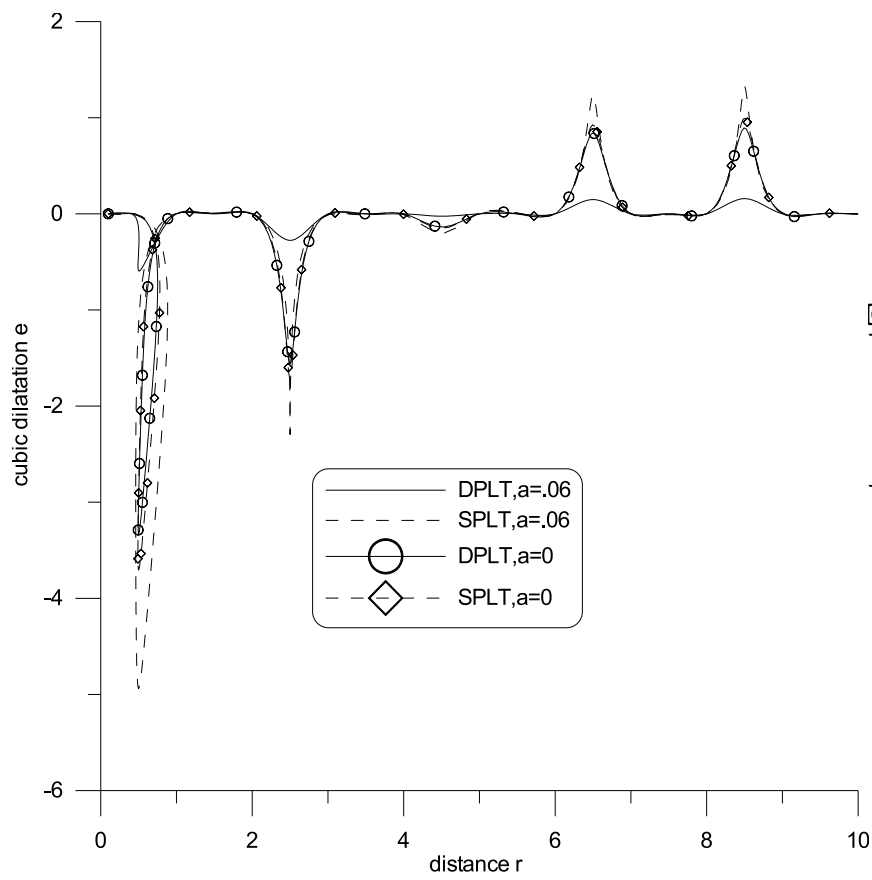

Fig. 7. Variations of cubic dilatation $e$ with distance $r$

3. The solid line with a centre symbol circle corresponds to dual-phase-lag of heat transfer without two temperature (DPLT, $a=0$ ).

4. The small dashed line with a centre symbol diamond corresponds to single-phase-lag of heat transfer with two temperature (SPLT, $a=0$ ).

Fig. 1. exhibits variations of displacement component $u_{r}$ with distance $r$. Here we notice that, away from the loading surface, DPLT and SPLT follow opposite oscillatory behaviour corresponding to both the cases, i.e. with two temperature and without two temperature. Fig. 2 shows variations of displacement component $u_{z}$ with distance $r$. It is noticed that variations of $u_{z}$ owing to DPLT corresponding to both the cases decrease as $r$ increases. Amplitude of oscillation corresponding to SPLT is greater as compared to DPLT; however, the behaviour is opposite oscillatory for the whole range corresponding to the models DPLT and SPLT. DPLT (SPLT) models, with two temperature and without two temperature, follow similar oscillatory trends with change in amplitude of oscillation. Fig. 3. shows Variation of stress component $\sigma_{z z}$ with distance $r$. We find that there is a sharp increase for the range $0 \leq r \leq 2$ in $\sigma_{z z}$ corresponding to DPLT in both the cases $a=0$ and $a=.06$ and trends are oscillatory with a decrease in amplitude. Corresponding to the case of SPLT, for $a=0$ and $a=.06$, trends are similar oscillatory with a difference in amplitude. However, DPLT and SPLT show opposite oscillatory trends. Fig. 4. gives variations of stress component $\sigma_{r z}$ with distance $r$. It is evident from this figure that there are small variations in $\sigma_{r z}$ corresponding to DPLT and SPLT for $a=.06$, whereas for $a=0$, the

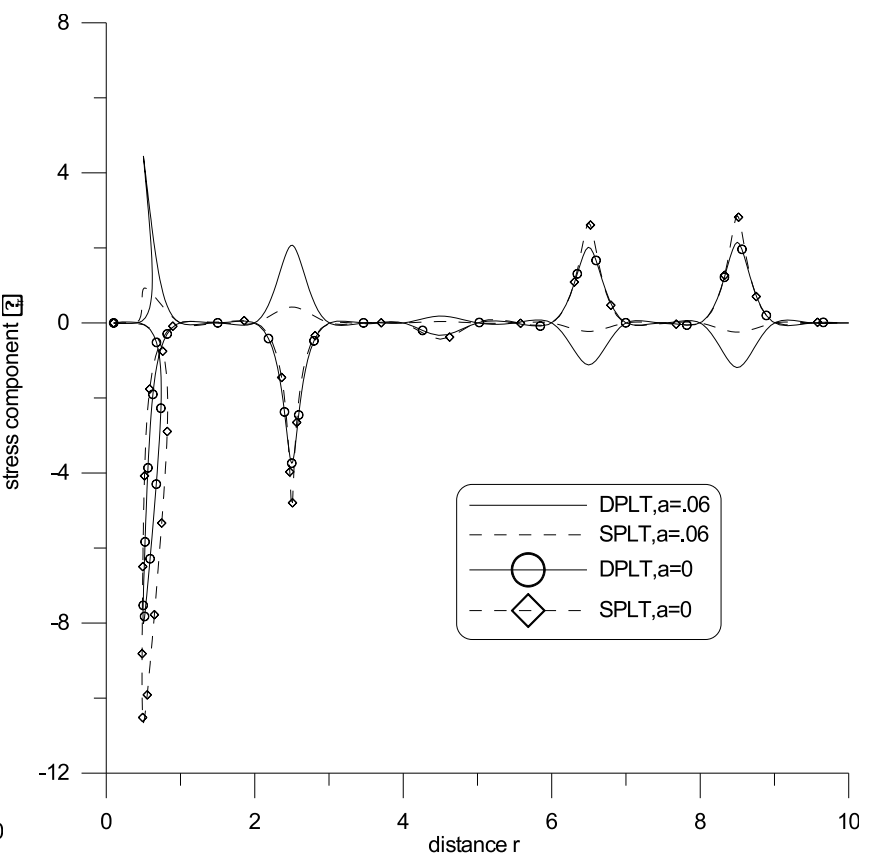

Fig. 8. Variations of stress component $\sigma_{r r}$ with distance $r$

pattern is opposite oscillatory. Fig. 5. gives variation of conductive temperature $\phi$ with distance $r$. Here in this case we notice that either there are sudden increases and decreases or there are small variations. Here descents are observed at the points $r=.5$ and $r=2.5$ and hikes are observed at the points $r=6.5$ and $r=9$. With two temperatures there are hikes and descents while without two temperature there are small variations. Fig. 6. exhibits Variations of temperature change $T$ with displacement $r$. Here there is a hike at the point $r=1$ and descents at the points $r=2.5, r=4.5$, $r=6.5$ and a small hike is observed at $r=9$ and small variations are observed for the remaining range except the small neighbourhoods of these points. Fig. 7 shows Variations of cubic dilatation $e$ with displacement $r$. Here, also either are small variations or sudden increase and decrease. Fig. 8 . exhibits variations of stress component $\sigma_{r r}$ with distance $r$. Here opposite trends are observed corresponding to the cases of without two temperature and with two temperature. As it is evident that without two temperature there is a descent at the point .5 whereas there is a hike at the same point in case of two temperature. While comparing the effect of phase lags, the trends are similar in both cases.

\section{CONCLUSION}

From the graphs it is evident that:

(i) There is a significant impact of two temperatures and phase lags (DPLT and SPLT ) on behaviour of deformation on various components of stresses, components of displacement, conductive temperature, temperature 
change and cubic dilatation in the ring.

(ii) $\operatorname{DPLT}(\mathrm{SPLT})$ for $a=0$ and $a=.06$ follow opposite oscillatory trends in case of $u_{r}, u_{z}, \sigma_{r z}, \sigma_{r r}$ and $\sigma_{z z}$. While from the graphs of $\phi, e, T$ the trends of DPLT and SPLT in both the cases $a=0$ and $a=.06$ are observed to be similar oscillatory.

(iii) We notice from $\sigma_{r r}, \phi, e, T$ that either the variations are very small or result in sudden hikes and dumps whereas in the rest of the cases variations move smoothly.

(iv) The use of thermal phase-lags in the heat conduction equation gives a more realistic model of thermoelastic media as it allows a delayed response to the relative heat flux vector.

The result of the problem is useful in the two dimensional problem of dynamic response due to various thermal and mechanical sources which has various geophysical and industrial applications.

\section{References}

[1] C. Catteno, A form of heat conduction equation which eliminates the paradox of instantaneous propagation, Compute Rendus 247, 431-433 (1958).

[2] P. Vernotte, Les paradox de la theorie continue de l'equation de la chaleur, Compute Rendus 246, 3145-3155 (1958).

[3] D.Y. Tzou, Macro to microscale Heat Transfer; The lagging behaviour, Taylor and Francis, Washington DC, USA 1996.

[4] R. Quintanilla, R. Racke, A note on stability in dual-phaselag heat conduction, International Journal of Heat and Mass Transfer 9(7-8), 1209-1213 (2006).

[5] R. Kumar, and S. Mukhopdhyay, Effects of thermal relaxation times on plane wave propagation under two temperature thermoelasticity, International Journal Of Engineering Sciences 48(2), 128-139 (2010).

[6] Y. Chou, R.J. Yang, Two dimensional dual-phase-lag thermal behaviour in single-/multi-layer structures using CESE method, International Journal of Heat and Mass Transfer 52, 239-249 (2009).

[7] J. Zhou, Y. Zang, J.K. Chen, An axisymmetric dual-phase-lag bioheat model for laser heating of living tissues, International Journal of Thermal Sciences 48, 1477-1485 (2009).

[8] R. Kumar, V. Chawla, I.A. Abbas, Effect of viscosity on wave propagation in anisotropic thermoelastic medium with threephase-lag model, Theoret. Appl. Mech 39(4), 313-341 (2012).

[9] X.H. Ying, J.X. Yun, Time fractional dual-phase-lag heat conduction equation, Chin. Phys. B 24(3), 034401 (2015).

[10] I.A. Abdallah, Dual phase lag Heat Conduction and Thermoelastic properties of a semi infinite medium Induced by Ultrashort Pulsed layer, Progress in Physics 3, 60-63 (2009).

[11] A.S. Rukolaine, Unphysical effects of the dual-phase-lag model of heat conduction, International Journal of Heat and Mass Transfer 78, 58-63 (2014).

[12] J.J Tripathi, G.D. Kedar, K.C. Deshmukh, Generalized thermoelastic diffusion problem in a thick circular plate with axisymmetric heat supply, Acta Mechanica 226(7), 2121-2134 (2015).

[13] P.J. Chen, M.E. Gurtin, On a theory of heat conduction involving two parameters, Zeitschrift für angewandte Mathematik und Physik (ZAMP) 19, 614-627 (1968).
[14] P.J. Chen, M.E. Gurtin, W.O. Williams, A note on simple heat conduction, Journal of Applied Mathematics and Physics (ZAMP) 19, 969-70 (1968).

[15] P.J. Chen, M.E. Gurtin, W.O. Williams, On the thermodynamics of non simple elastic materials with two temperatures, ZAMP 20, 107-112 (1969).

[16] B.A. Boley, I.S. Tolins, Transient coupled thermoelastic boundary value problem in the half space, Journal of Applied Mechanics 29, 637-646 (1962).

[17] W.E. Warren, P.J. Chen, Wave propagation in the two temperature theory of thermoelasticity, Journal of Acta Mechanica 16, 21-33 (1973).

[18] Youssef, H.M. Theory of two - temperature thermoelasticity without energy dissipation, Journal of Thermal Stresses, 34, 138-146. (2011)

[19] I.A. Abbas, R. Kumar, L.S. Reen, Response of thermal sources in transversely isotropic thermoelastic materials without energy dissipation and with two temperatures, Canadian Journal of Physics 92(11), 1305-11 (2014).

[20] R. Kumar, S. Mukhopadhyay, Analysis of the Effects of Phaselags on propagation of harmonic Plane Waves in Thermoelastic Media, CMST 16(1), 19-28 (2010).

[21] H.M. Youssef, Theory of two temperature generalized thermoelasticity, IMA Journal of Applied Mathematics 71(3), 383390 (2006).

[22] R. Kumar, K.D. Sharma, S.K. Garg, Effect of two temperature on reflection coefficient in micropolar thermoelastic media with and without energy dissipation, Advances in Acoustics and Vibrations (2014).

[23] S. Kaushal, R. Kumar, A. Miglani, Wave propagation in temperature rate dependent thermoelasticity with two temperatures, Mathematical Sciences 5, 125-146 (2011).

[24] S. Kaushal, N. Sharma, R. Kumar, Propagation of waves in generalized thermoelastic continua with two temperature, International Journal of Applied Mechanics and Engineering 15, 1111-1127 (2010).

[25] M.A. Ezzat, E.S. Awad, Constitutive relations, uniqueness of solution and thermal shock application in the linear theory of micropolar generalized thermoelasticity involving two temperatures, Journal of Thermal Stresses 33(3), 225-250 (2010).

[26] K. Sharma, M. Marin, Effect of distinct conductive and thermodynamic temperatures on the reflection of plane waves in micropolar elastic half-space, U.P.B.Sci.Bull Series 75(2), 121-132 (2013).

[27] S.Y. Atwa, A. Jahangir, Two temperature effects on plane waves in generalized Thermo-Microstretch Elastic Solid, International Journal of Thermophysics 35,175-193 (2014).

[28] S. Mondal, S.H. Malik, S. Kanoria, Fractional order two temperature dual phase lag thermoelasticity with variable thermal conductivity, International Scholarly Research Notices, 646049 (2014).

[29] S.M. Said, Deformation of a rotating two temperature generalized magneto thermoelastic medium with initial heat source due to hydrostatic initial stress, Meccanica 50(8), 2077-2091 (2015).

[30] S.M. Said, Two-temperature generalized magnetothermoelastic medium for dual-phase-lag model under the effect of gravity field and hydrostatic initial stress, Multidiscipline Modeling in Materials and Structures 12(2), 362-383 (2016)

[31] K. Lofty, Elastic wave motions for a photothermal medium of a dual phase lag model with an internal heat source and gravitational field, Canadian Journal of Physicsl 94(4), 400-409 (2016).

[32] D.P. Gaver, Observing stochastic processes and approximate transform inversion, Operations Res 14, 444-459 (1966). 
[33] H. Stehfast, Algorithm 368, Numerical inversion of Laplace Transforms, Comm. Ass'n. Mach, 13, pp.47-49 (1970).

[34] H. Stehfast; Remark on Algorithm 368, Numerical inversion of Laplace Transforms, Comm. Ass'n. Mach, 3, 624 (1970).
[35] W.H. Press, B.P. Flannery, S.A. Teukolsky, W.A. Vatterling, Numerical recipes, Cambridge University Press, Cambridge, the art of scientific computing 1986.

[36] R.S. Dhaliwal, A. Singh, Dynamic coupled thermoelasticity, Hindustance Publisher Corp., New Delhi India, 1980.
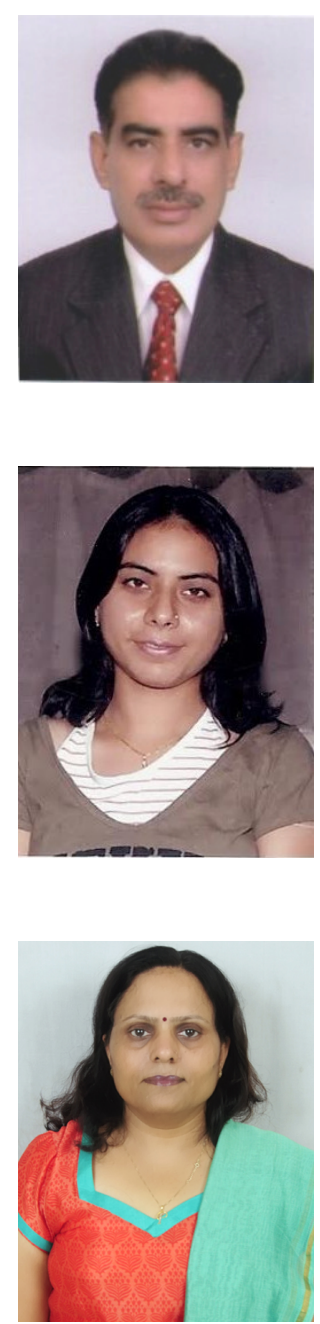

Rajneesh Kumar. Prof. Rajneesh Kumar is Professor in Mathematics at Kurukshetra University, Kurukshetra. His field of expertise is Solid Mechanics and Thermoelasticity.

Nidhi Sharma. Dr Nidhi Sharma is an Associate Professor in Mathematics in the department of Mathematics, MM University Mullana. Her field of research is thermoelasticity.

Parveen Lata. The corresponding author Parveen Lata is Assistant Professor in Mathematics in the Department of Basic and Applied Sciences, Punjabi University Patiala. She is working on thermoelasticity in collaboration with Prof. Rajneesh Kumar and Dr Nidhi Sharma. Recently they have published various papers on thermoelasticity with two temperatures and without energy dissipation. 